\title{
High Efficiency Electron Diffractive Optics
}

\author{
Jordan S. Pierce, Tyler R. Harvey, Tyler S. Yahn, Benjamin J. McMorran \\ Department of Physics, University of Oregon, Eugene, OR 97405, USA
}

We have utilized FIB milling to create nanoscale phase gratings for producing predetermined electron wavefronts in the diffracted orders. Electron beams with specifically manufactured wavefronts are being studied for various potential applications. Many of these applications utilize only the first order diffracted beams and try to reduce the background noise produced by the zeroth order beam, which is usually much more intense than the diffracted beams. We are particularly interested in creating electron vortex beams to study the interaction of the OAM beam with matter.

Electron vortex beams have applications in high resolution dichroism [2] for magnetic domain imaging. For this and for most applications, the use of electron vortex beams requires diffractive optics (holograms) with high efficiency in the first diffraction order, since the zeroth order beam is usually either unnecessary or adds unwanted background noise. Using nanoscale diffraction gratings, laguerre-gaussian beams carrying up to $100 \hbar$ of orbital angular momentum have been created [1]. We have demonstrated phase grating with efficiency up to $150 \%$.

All patterns and phase gratings used in this analysis were created using an FEI Helios NanoLab 600. In order to increase diffraction efficiency, we have varied many parameters, such as ion beam current, ion beam dwell, milling method, pattern type, membrane thickness, and material. Potential problems encountered while milling are membrane stretch and thermal drift, both of which lead to a loss of efficiency and to a loss of spatial coherence of the diffraction pattern. Depending on the size of the grating to be milled and the limitation of the FIB used, these problems can be greatly diminished and high efficiency beams can be created. By varying these parameters, we can create sinusoidal phase gratings that impart close to a $\pi$ phase shift to a $300 \mathrm{keV}$ electron beam, creating high efficiency diffracted orders.

An example of a high efficiency phase grating with a fork dislocation is shown in Figure 2, with the corresponding diffraction pattern shown beneath it, and the relative intensities are shown in Figure 1. Here the hologram pattern is 5 microns wide on a $50 \mathrm{~nm}$ thick silicon nitride membrane. The diffraction pattern was taken it a TEM at $300 \mathrm{keV}$. The relative efficiency of the grating can be tuned so that, if necessary, the intensities in the first diffraction orders and the zeroth order are equal, as shown in Figure 3. Figures 4 and 5 show the surface profiles for these two holograms, showing a feature depth of more than $25 \mathrm{~nm}$.

We have used this fabrication technique to produce diffractive electron optics with down to $40 \mathrm{~nm}$ linewidth spacing, yielding free space diffraction angles up to $49 \mu \mathrm{rad}$, covering up to $\oslash 80 \mu \mathrm{m}$ areas, and with high spatial coherence. High diffraction efficiency gratings have been produced in which intensity in the first diffracted orders are $150 \%$ the intensity of $0^{\text {th }}$ order. These parameters open the door to high-resolution TEM studies using electron vortex beams.

References

[1] B. J. McMorran et al., Science 331 (2011) 192.

[2] J. C. Idrobo and S. J Pennycook, Journal of Electron Microscopy 60(5): 295-300 (2011) 


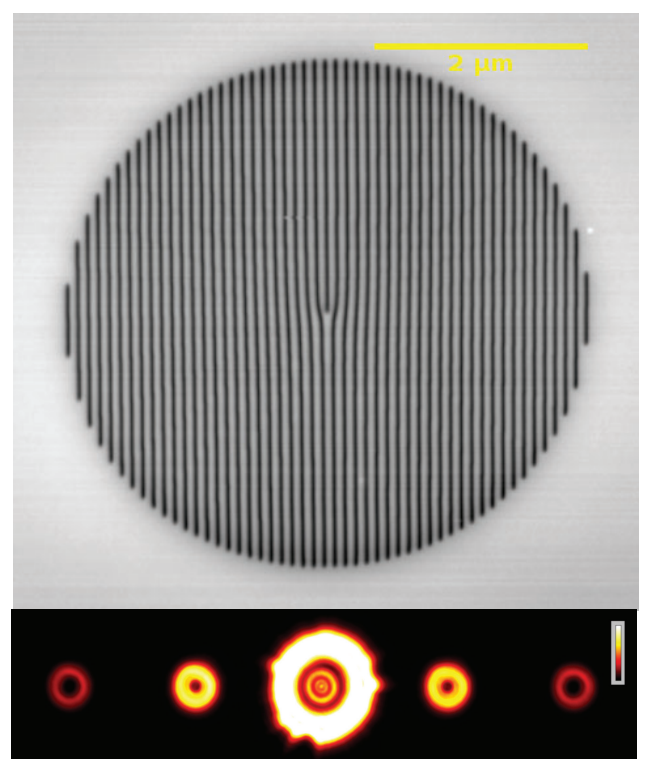

Figure 2: AFM raster of a high efficiency fork dislocation hologram in a $50 \mathrm{~nm}$ silicon nitride membrane together with the diffracted image at $300 \mathrm{keV}$. Here the aperture is larger than the grating, resulting in excess intensity in the $0^{\text {th }}$ order.

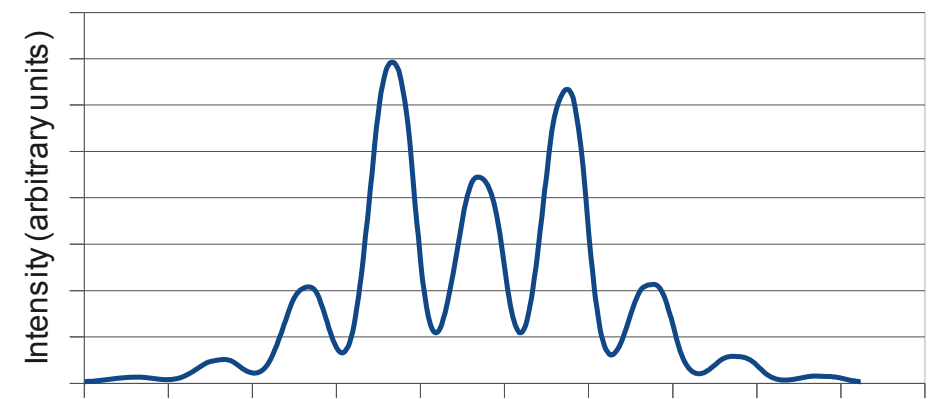

Figure 1: Intensity of the diffracted beams for the phase grating in Figure 2. This data is not from the diffraction image in Figure 2, but it is from the same grating.

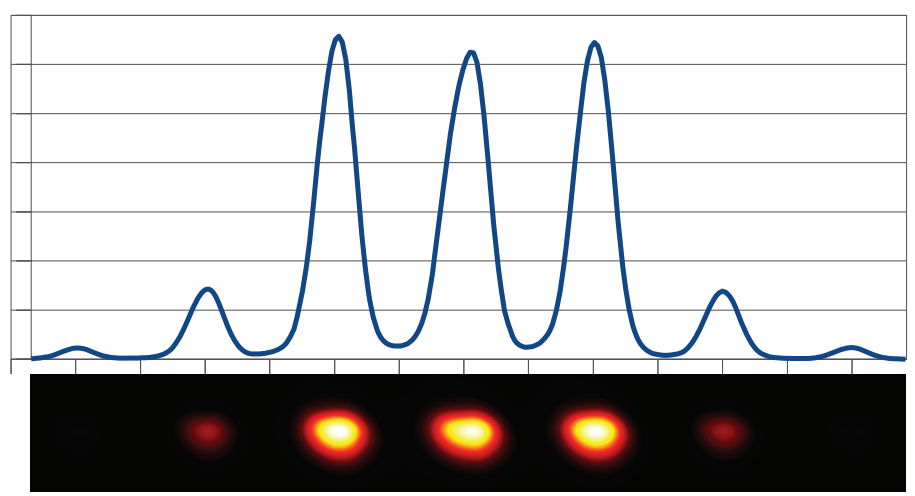

Figure 3: Diffraction of a high efficiency phase grating milled with optimum parameters.

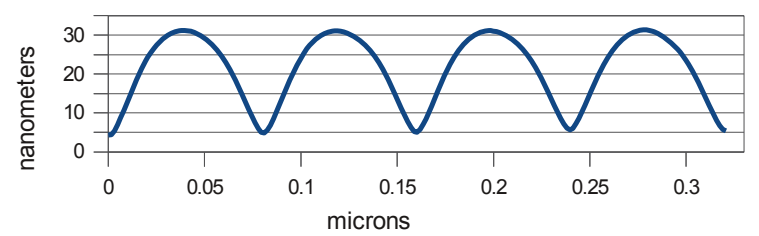

Figure 4: Profile of a section of the grating in Figure 2. Peak to valley distance is about 28 nm.

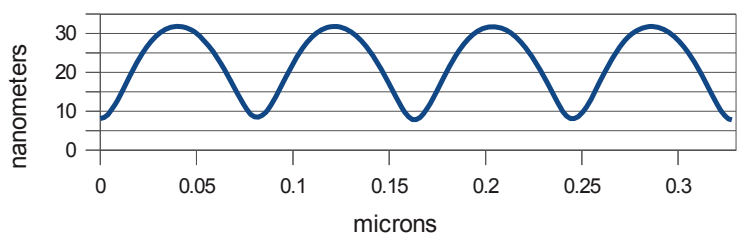

Figure 5: Profile of a section of the grating used in Figure 3. Peak to valley distance is about $24.2 \mathrm{~nm}$ 